Research Article

\title{
An Approach to Determining Attribute Weights Based on Integrating Preference Information on Attributes with Decision Matrix
}

\author{
Quan Zhang ${ }^{1}{ }^{1}$ and HongWei Xiu ${ }^{2}$ \\ ${ }^{1}$ School of Information Engineering, Shenyang University of Technology, Shenyang, China \\ ${ }^{2}$ Alumni Association, Shenyang Jianzhu University, Shenyang, China \\ Correspondence should be addressed to Quan Zhang; isqzhang@sut.edu.cn
}

Received 25 May 2018; Revised 15 July 2018; Accepted 29 August 2018; Published 1 October 2018

Academic Editor: Daniele Bibbo

Copyright (c) 2018 Quan Zhang and HongWei Xiu. This is an open access article distributed under the Creative Commons Attribution License, which permits unrestricted use, distribution, and reproduction in any medium, provided the original work is properly cited.

\begin{abstract}
The interval multiple attribute decision-making problems are studied in this paper, where the preference information on attributes is expressed with preference orderings, linguistic terms, interval numbers, and inequality constraints among partial attribute weights. An approach is proposed to determine the attribute weights based on the preference information on attributes and the interval decision matrix. Firstly, preference orderings, linguistic terms, and interval numbers are normalized and aggregated into the group opinions, based on which an optimization model is set up to calculate the subjective attribute weights by including inequality constraints among partial attribute weights in the model. Then, based on the interval decision matrix, the entropy method is adopted to calculate the objective attribute weights, which is integrated with the subjective weights so that both the subjective preference information and the objective information in the decision matrix are reflected. Finally, an example is used to illustrate the proposed approach.
\end{abstract}

\section{Introduction}

Multiple attribute decision making (MADM) refers to ranking or selecting the alternatives that are associated with noncommensurate and conflicting attributes [1-3]. In the course of multiple attribute decision making, experts are usually invited to help making decisions by providing their preference information against attributes or alternatives $[4,5]$. The preference given by experts belongs to the subjective information $[6,7]$, which can be used to calculate the attribute weights. Given a decision matrix, the attribute weights decide alternative rankings or the best choice among them $[8,9]$. While determining the attribute weights, commonly, there are two types of methods: the methods based on the subjective information $[8,9]$ (that is called the subjective method in this paper) and those based on the decision matrix (that is called the objective method in this paper).

On the one hand, if only subjective information is used for determining the attribute weights, it would result in prejudice or deviation from the objective facts because it only reflects experts' subjective opinions $[6,10]$. On the other hand, the attribute weights can also be determined based on the decision matrix. The attribute values of alternatives in the decision matrix are considered as the objective information $[3,6]$. If the attribute weights are determined only based on the decision matrix, they would be meaningless because of neglecting the experts' subjective information [11]. Thus, in the course of determining attribute weights, both the subjective method and the objective method have their limitations, respectively. How to combine these two types of methods to reflect both the experts' subjective preference information and the objective information in decision matrix is a problem worth studying [11-14].

This paper focuses on determining the attribute weights based on experts' subjective preference information and the interval decision matrix. The experts' subjective preference information plays important roles in determining the attribute weights $[4,7]$. It is noticed that, in fuzzy or uncertain 
environment, it is difficult to obtain precise decision information [15]. Especially, when the experts involved have different culture and education backgrounds, they often intend to present their subjective preference information on the attribute weights by means of their most easy ways, for example, preference orderings [16], linguistic terms $[3,13,17]$, interval numbers [7], or inequality constraints among the partial attribute weights $[18,19]$.

Integration of decision information is desirable for solving uncertain MADM problems [20]. Currently, there is seldom research on integrating the decision matrix with the experts' subjective preference information on attribute weights [21]. In [22], the auctioneer provides preference on attribute weights, as well as on attribute values and alternatives. The preference on attribute weights is presented with the form of linear inequalities. In [23], the attribute values in decision matrix are presented with crisp values, fuzzy numbers, interval numbers, and linguistic terms. Two types of subjective attribute weights are provided: multiplicative preference relations and fuzzy preference relations.

However, in fuzzy or uncertain decision making environment, for example, the assessment of international cooperation projects across different countries and regions, the experts are invited from different countries and regions and usually have difficulty in giving precise preference information on the attributes, for example, multiplicative preference relations and fuzzy preference relations. In addition, the experts would like to use easier ways to give their preference information on the attribute weights, such as preference orderings, linguistic terms, interval numbers, and inequality constraints among the attribute weights. Instead of utility values, preference orderings are easy ways for experts to describe the relative importance of attributes when they can only give rankings of the attributes. Linguistic terms are natural ways for experts to present their preference information, which can reduce the burden for them to express their opinions. Interval numbers are also the easy ways for experts to present their preference information when they could not give exact values of the attribute weights. Inequality constraints among the attribute weights usually describe the relative comparisons among partial attributes, for example, one attribute is more important than another one.

It is desirable to deal with preference orderings, linguistic terms, interval numbers, and inequality constraints among the attribute weights in the MADM process since they are the common ways for experts to use when expressing their subjective preference on attributes easily and accurately. Furthermore, it is desirable to determine the attribute weights by integrating the interval decision matrix with experts' preference information on attribute weights in the forms of preference orderings, linguistic terms, interval numbers, and inequality constraints among the attribute weights.

The purpose of this paper is to propose an approach to determining attribute weights based on integrating interval decision matrix and the experts' preference information on attributes in the formats of preference orderings, linguistic terms, interval numbers, and inequality constraints among them. The organization of this paper is as follows: the MADM problem with interval decision matrix and experts' preference information on attributes is described in Section 2. Section 3 proposes the approach to determining the attribute weights by addressing the normalization and aggregation process for the preference information and setting up an integrating optimization model. In Section 4, an example is used to illustrate the proposed approach. Summary is given in Section 5.

\section{Problem Descriptions}

In order to facilitate describing the MADM problem with interval decision matrix and experts' preference information on attributes in the formats of preference orderings, linguistic terms, interval numbers, and inequality constraints among the attribute weights, the following assumptions and notations are adopted:

let $S=\left\{S_{1}, S_{2}, \ldots, S_{m}\right\}$ denote a discrete set of $m(\geq 2)$ possible alternatives;

let $C=\left\{c_{1}, c_{2}, \ldots, c_{n}\right\}$ denote a set of $n(\geq 2)$ attributes;

let $W=\left(w_{1}, w_{2}, \ldots, w_{n}\right)$ denote the vector of $n(\geq 2)$ attribute weights, where $w_{j}$ is the weight of attribute $c_{j}$, while $\sum_{j=1}^{n} w_{j}=1$ and $w_{j} \geq 0$ holds for $j=1, \ldots, n$.

Let $\widetilde{A}=\left[\widetilde{a}_{i j}\right]$ denote the interval decision matrix, where, $\tilde{a}_{i j}=\left[a_{i j}^{L}, a_{i j}^{U}\right]$ is the interval consequence for alternative $\tilde{q}_{i j}$ with respect to attribute $c_{j}, B=\left[b_{i j}\right]_{m \times n}, j=1, \ldots, n$.

The experts involved are known: let $E=\left\{e_{1}, e_{2}, \ldots, e_{K}\right\}$ $(k \geq 2)$ denote the set of experts. Different experts express their subjective preference information on attributes in the following formats, i.e., preference orderings, linguistic terms, interval numbers, and inequality constraints among the attribute weights, as stated in Table 1.

The problem left is to propose an approach to determining the attribute weights based on integrating the interval decision matrix and the experts' preference information on attributes in the formats of preference orderings, linguistic terms, interval numbers, and inequality constraints among them, as stated in Table 1.

\section{The Proposed Approach}

3.1. Determining Attribute Weights Based on the Experts' Preference Information. As stated in Table 1, in this paper, the experts express their subjective preference information on attributes in the formats of preference orderings, linguistic terms, interval numbers, and inequality constraints among them. In order to determine the attribute weights based on the experts' preference information, normalization and aggregation would be employed. Based on the aggregation results, preference exploitation would be conducted by setting up an optimization model including the inequality constraints among the attribute weights.

In order to facilitate describing the proposed approach, the definition of fuzzy preference relation is given firstly.

Definition 1. A fuzzy preference relation on the attributes is a binary fuzzy relation $P$ on $C$, where $P$ is a mapping $C \times$ $C \longrightarrow[0,1]$ and $p_{j r}$ denotes the preference degree of attribute $c_{j}$ over $c_{r}$. It is assumed that $P$ is reciprocal, by definition, (i) $p_{j r}+p_{r j}=1$ and (ii) $p_{j j}=-$ (symbol "-" 
TABLE 1: Experts' subjective preference information on attributes.

\begin{tabular}{lcccc}
\hline & $c_{1}$ & $c_{2}$ & $c_{3}$ & $c_{4}$ \\
\hline$e_{1}$ (preference orderings) & 3 & 2 & 4 & 1 \\
$e_{2}$ (linguistic terms) & Fair & Important & Unimportant & Very important \\
$e_{3}$ (interval numbers) & {$[0.10,0.30]$} & {$[0.15,0.35]$} & {$[0.15,0.20]$} & {$[0.25,0.40]$} \\
$e_{4}$ (inequality constraints among attribute weights) & & & $w_{2}>w_{3}$ & \\
\hline
\end{tabular}

means that there is no need to give any preference information on attribute $c_{j}$ ).

3.1.1. Normalize Experts' Preference Information. To normalize the experts' preference information on attributes in the formats of preference orderings, linguistic terms, and interval numbers, the definition of linguistic terms is presented as follows, as well as the basic linguistic evaluation set is addressed.

Definition 2. A linguistic term $\widetilde{T}$ on real number set is defined as a triangular fuzzy number (denoted as $(u, \alpha, \beta)$ ), if its membership function $\mu_{\widetilde{T}}\left(R^{+} \longrightarrow[0,1]\right)$ is defined as,

$$
\mu_{\widetilde{T}}(x)= \begin{cases}\frac{x-\alpha}{u-\alpha}, & x \in[\alpha, u], \\ \frac{x-\beta}{u-\beta}, & x \in[u, \beta], \\ 0, & \text { otherwise, }\end{cases}
$$

where $\alpha \leq u \leq \beta$, and $u$ is the model value and $\alpha$ and $\beta$ stand for the lower value and the upper value of linguistic term $\widetilde{T}$, respectively.

A linguistic term set $\left\{t_{0}, t_{1}, \ldots, t_{g}\right\}$ is an ordering set, which is composed of a number of linguistic terms with odd number (i.e., $g+1$ is the odd number), for example, a set of seven terms, i.e., $\left\{t_{0}=\right.$ "none," $t_{1}=$ "very poor," $t_{2}=$ "poor" $t_{3}=$ "fair," $t_{4}=$ "good," $t_{5}=$ "very good," amd $t_{6}=$ "perfect" $\}$. The following properties of linguistic term set $\left\{t_{0}, t_{1}, \ldots, t_{g}\right\}$ are assumed [7, 24]: (1) it is ordered: $t_{i} \geq t_{j}$, if $i \geq j$. The symbol " $\geq$ " denotes "better or equal"; (2) there is the negation operator "Neg": Neg $\left(t_{i}\right)=t_{j}$, such that $j=g-i$, where $g+1$ is the number of elements in $\left\{t_{0}, t_{1}, \ldots, t_{g}\right\}$, and the largest term is $t_{g}$; (3) There is the max operator and the min operator: $\operatorname{Max}\left\{t_{i}, t_{j}\right\}=t_{i}$ and $\operatorname{Min}\left\{t_{i}, t_{j}\right\}=t_{j}$ if $t_{i} \geq t_{j}$.

Since different experts would use different linguistic term sets when expressing their subjective preference information against the attributes, the linguistic term sets would be of different granularity. A basic linguistic evaluation term set would be used for normalizing the linguistic terms with different granularity. In this paper, the basic linguistic evaluation term set TERMSET is defined as $\left\{\right.$ term $_{0}$, term $_{1}, \ldots$, term $\left.{ }_{g}\right\}$, where $g+1$ is the odd number. The membership functions (including the model value $u_{l}$, the lower value $\alpha_{l}$, and the upper value $\beta_{l}$ ) of triangular fuzzy numbers $\gamma_{l}=\left(u_{l}, \alpha_{l}, \beta_{l}\right)$ for the elements in the basic linguistic evaluation set (i.e., TERMSET $=\left\{\operatorname{term}_{0}, \operatorname{term}_{1}, \ldots\right.$, term $\left._{g}\right\}$ ) are defined as in $(2), l=0,1, \ldots, g$.

$$
\gamma_{l}= \begin{cases}\alpha_{0}=0, & \\ u_{l}=\frac{l}{g-1}, & 0 \leq l \leq g-1, \\ \alpha_{l}=\frac{l-1}{g-1}, & 1 \leq l \leq g-1, \\ \beta_{l}=\frac{l+1}{g-1}, & 0 \leq l \leq g-2, \\ \beta_{g-1}=1, & \end{cases}
$$

where $g+1$ is the number of terms in the basic linguistic evaluation set TERMSET.

In this paper, \{"less important," "unimportant," "fair," "important," and "very important" $\}$ is adopted as the basic linguistic evaluation set TERMSET.

(1) Transform preference orderings into fuzzy preference relations

Given preference orderings $O^{k}=\left(o_{1}^{k}, o_{2}^{k}, \ldots, o_{n}^{k}\right)$ by expert $e_{k}(k=1, \ldots, K)$, the following steps can be used to transform $O^{k}=\left(o_{1}^{k}, o_{2}^{k}, \ldots, o_{n}^{k}\right)$ into the fuzzy preference relations on attributes.

Step 1. Set up the corresponding intervals for $n$ ranking positions, inter $_{j}=[(n-j / n),(n+1-j / n)], 1 \leq j \leq n$.

Step 2. Set up the membership functions for the corresponding interval of the ranking positions, i.e.,

$$
y(j)= \begin{cases}1, & \frac{n-j}{n} \leq x \leq \frac{n+1-j}{n}, \\ 0, & \text { others. }\end{cases}
$$

Thus, the preference ordering of attribute $c_{j}$ can be transformed into a subset in $[0,1]$.

Step 3. Based on the interval membership functions corresponding to the ranking positions of attribute $c_{j}$, the following definition 3 can be used to transform inter into $_{j}$ a fuzzy set over the basic linguistic evaluation set TERMSET, $j=1, \ldots, n$.

Definition 3. Suppose $\tilde{\lambda}$ is an interval and TERMSET $=$

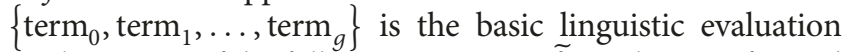
set, by means of the following mapping, $\tilde{\lambda}$ can be transformed into a fuzzy set over TERMSET, 


$$
\tau: \tilde{\lambda} \longrightarrow F(\text { TERMSET }) .
$$

Formula (4) can be rewritten as the form of fuzzy set over TERMSET,

$$
\tau(\widetilde{\lambda})=\left\{\left(\operatorname{term}_{l}, \gamma_{l}\right) \mid l \in[0, g]\right\},
$$

where

$$
\gamma_{l}=\max _{y} \min \left\{\mu_{\widetilde{\lambda}}(y), \mu_{\mathrm{term}_{l}}(y)\right\},
$$

where $\mu_{\tilde{\lambda}}(y)$ and $\mu_{\text {term }_{l}}(y)$ are the membership functions of

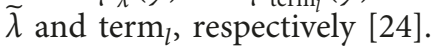

Denote $\tau\left(o_{j}^{k}\right)=F_{j}^{k}$ (TERMSET) as the fuzzy set over TERMSET, for the ranking position $o_{j}^{k}, j=1, \ldots, n$.

Step 4. Transform $F_{j}^{k}$ (TERMSET) into the fuzzy preference relations on the attributes.
Suppose $F_{j}^{k}($ TERMSET $)=\left\{\left(\right.\right.$ term $\left._{0}, \gamma_{j, 0}^{k}\right),\left(\right.$ term $\left._{1}, \gamma_{j, 1}^{k}\right), \ldots$ (term $\left.\left._{g}, \gamma_{j, g}^{k}\right)\right\}$ is the fuzzy set over TERMSET for the ranking position $o_{j}^{k}, j=1, \ldots, n$, which is obtained in Step 3. The following mapping $\chi$ in (7) can be used to transform $F_{j}^{k}$ (TERMSET) into a crisp value:

$$
\chi: F_{j}^{k}(\text { TERMSET }) \longrightarrow[0, g],
$$

where formula (7) is stated as

$$
\chi\left(F_{j}^{k}(\text { TERMSET })\right)=\frac{\sum_{l=0}^{g} \gamma_{j, l}^{k} \cdot l}{g \sum_{l=0}^{g} \gamma_{j, l}^{k}} .
$$

Therefore, given the preference orderings $O^{k}=\left(o_{1}^{k}\right.$, $\left.o_{2}^{k}, \ldots, o_{n}^{k}\right)$ by expert $e_{k}(k=1, \ldots, K)$, the fuzzy preference relations between $c_{j}$ and $c_{r}$ can be obtained as in the following equation:

$$
p_{j r}^{k}=\frac{\left.\chi\left(F_{j}^{k} \text { TERMSET }\right)\right)}{\chi\left(F_{j}^{k}(\text { TERMSET })\right)+\chi\left(F_{r}^{k}(\text { TERMSET })\right)}, \quad j, r=1, \ldots, n
$$

(2) Transform linguistic terms into fuzzy preference relations

Suppose expert $e_{k}(k=1, \ldots, K)$ gives his/her preference against the attributes with a linguistic term vector ling $^{k}=\left(\operatorname{ling}_{1}^{k}, \ldots\right.$, ling $\left._{n}^{k}\right)$, and ling ${ }_{j}^{k}$ is the linguistic term for attribute $c_{j}$, then the following method is used to transform $\operatorname{ling}_{j}^{k}$ into the fuzzy set over TERMSET:

$$
\tau\left(\operatorname{ling}_{j}^{k}\right)=\left(\operatorname{term}_{l}, \gamma_{j, l}^{k}\right) \mid l \in[0, g], \quad j=1, \ldots n,
$$

where

$$
\gamma_{j, l}^{k}=\max _{y} \min \left\{\mu_{\operatorname{ling}_{j}^{k}}(y), \mu_{\operatorname{term}_{l}}(y)\right\},
$$

where $\mu_{\operatorname{ling}^{k}}(y)$ and $\mu_{\text {term }}(y)$ are the membership functions of $\operatorname{ling}_{j}^{k}$ and term ${ }_{l}$, respectively.

Therefore, given the preference information of linguistic term vector ling ${ }^{k}=\left(\operatorname{ling}_{1}^{k}, \ldots\right.$, ling $\left._{n}^{k}\right)$ from expert $e_{k}(k=$
$1, \ldots, K)$, the fuzzy preference relation between $c_{j}$ and $c_{r}$ is obtained as follows:

$$
p_{j r}^{k}=\frac{\chi\left(\tau\left(\operatorname{ling}_{j}^{k}\right)\right)}{\chi\left(\tau\left(\operatorname{ling}_{j}^{k}\right)\right)+\chi\left(\tau\left(\operatorname{ling}_{r}^{k}\right)\right)} \quad j, r=1, \ldots, n,
$$

where

$$
\chi\left(\tau\left(\operatorname{ling}_{j}^{k}\right)\right)=\frac{\sum_{l=0}^{g} \gamma_{j, l}^{k} \cdot l}{\sum_{l=0}^{g} \gamma_{j, l}^{k}}
$$

(3) Transform interval numbers into fuzzy preference relations

Suppose expert $e_{k}(k=1, \ldots, K)$ gives his/her preference information on the attributes in the format of interval numbers, for example, ineral ${ }_{j}^{k}=\left[\operatorname{ineral}_{j}^{k L}\right.$, ineral $\left._{j}^{k U}\right]$ is for attribute $c_{j}$ and ineral ${ }_{r}^{k}=\left[\right.$ ineral $_{r}^{k L}$, ineral $\left.{ }_{r}^{k U}\right]$ is for attribute $c_{r}$, then, the fuzzy preference relations between $c_{j}$ and $c_{r}$ is obtained as follows [25]:

$$
p_{j r}^{k}=\frac{\max \left\{0, \text { len }\left(\text { ineral }_{j}^{k}\right)+\operatorname{len}\left(\text { ineral }_{r}^{k}\right)-\max \left(\text { ineral }_{r}^{k U}-\text { ineral }_{j}^{k L}, 0\right)\right\}}{\operatorname{len}\left(\text { ineral }_{j}^{k}\right)+\operatorname{len}\left(\text { ineral }_{r}^{k}\right)}, j, r=1, \ldots, n,
$$

where len $\left(\right.$ ineral $\left._{j}^{k}\right)$ and len $\left(\right.$ ineral $\left._{r}^{k}\right)$ are the lengths of ineral ${ }_{j}^{k}$ and ineral ${ }_{r}^{k}$, respectively.

$$
\begin{aligned}
\operatorname{len}\left(\operatorname{ineral}_{j}^{k}\right) & =\operatorname{ineral}_{j}^{k U}-\text { ineral }_{j}^{k L}, \\
\operatorname{len}\left(\operatorname{ineral}_{r}^{k}\right) & =\text { ineral }_{r}^{k U}-\text { ineral }_{r}^{k L} .
\end{aligned}
$$

3.1.2. Aggregate Experts' Preference Information. After normalizing the experts' subjective preference information on the attributes in 3.1.1, their preference information are transformed into the fuzzy preference relations, respectively, and the next step is to aggregate these resulting fuzzy preference relations. In this paper, the "simple additive 
weighting method" is used to aggregate these resulting fuzzy preference relations from the experts, i.e.,

$$
p_{j r}=\sum_{k=1}^{K} \lambda_{k} \cdot p_{j r}^{k}, \quad k=1, \ldots, K, j, r=1, \ldots, n,
$$

where $p_{j r}^{k}$ is the fuzzy preference relations between attributes $c_{j}$ and $c_{r}$, which is derived from expert $e_{k}$ 's preference information, $k=1, \ldots, K, j, r=1, \ldots, n$. $\lambda_{k}$ is the weight of expert $e_{k}$.

Denote the group fuzzy preference relations as $P=\left(p_{j r}\right)_{n \times n}$, where $p_{j r}$ is obtained in (16).

3.1.3. Determine Attribute Weights Based on the Experts' Preference Information. Given the group fuzzy preference relations $P=\left(p_{j r}\right)_{n \times n}$ obtained in (16), $p_{j r}$ should be as close as possible with $w_{j} /\left(w_{j}+w_{r}\right)$. It is noticed that $p_{j r}+p_{r j}=1$, and therefore, the following optimization model can be set up to determine the attribute weights based on the group fuzzy preference relations $P=\left(p_{j r}\right)_{n \times n}$ :

$$
\min Z=\sum_{j=1}^{n} \sum_{r=1}^{n}\left(p_{j r} w_{r}-p_{r j} w_{j}\right)^{2},
$$

such that

$$
\begin{aligned}
\sum_{j=1}^{n} w_{j} & =1, \\
w_{j} & >0, \quad j=1, \ldots, n .
\end{aligned}
$$

If the inequality constraints among attributes are considered, for example, $w_{2}>w_{3}$, model (17a)-(17c) would be modified into the following one:

$$
\min Z=\sum_{j=1}^{n} \sum_{r=1}^{n}\left(p_{j r} w_{r}-p_{r j} w_{j}\right)^{2},
$$

such that

$$
\begin{gathered}
\sum_{j=1}^{n} w_{j}=1, \\
w_{2}>w_{3}, \\
w_{j} \geq 0, \quad j=1, \ldots, n,
\end{gathered}
$$

where formula (18c) denotes that attribute $c_{2}$ is more important than $c_{3}$ (i.e., $w_{2}>w_{3}$ ).

Models (18a)-(18d) can be solved by means of Matlab Toolbox. Denote the attribute weight vector obtained by solving Models (18a) $-(18 \mathrm{~d})$ as $w^{\prime}=\left(w_{1}^{\prime}, w_{2}^{\prime}, \ldots, w_{n}^{\prime}\right)$, which is derived from the experts' subjective preference information in different formats as stated above.

3.2. Determine Attribute Weights Based on the Interval Decision Matrix. Given the interval decision matrix $\widetilde{A}=$ $\left[\widetilde{a}_{i j}\right]_{m \times n}$, suppose it has been normalized into the beneficial and dimensionless one $\widetilde{Q}=\left[\widetilde{q}_{i j}\right]_{m \times n}[26] . \widetilde{Q}=\left[\widetilde{q}_{i j}\right]_{m \times n}$ can be transformed into a single-point one by means of the following operations:

Definition 4. Given the beneficial and dimensionless interval decision matrix $\widetilde{Q}=\left[\widetilde{q}_{i j}\right]_{m \times n}\left(\widetilde{q}_{i j}=\left[q_{i j}^{L}, q_{i j}^{U}\right]\right)$, the base interval for attribute $c_{j}$ is defined as

$$
\operatorname{pos}_{j}^{+}=\left[\operatorname{pos}_{j}^{+L}, \operatorname{pos}_{j}^{+U}\right], \quad j=1, \ldots, n,
$$

where

$$
\begin{aligned}
& \operatorname{pos}_{j}^{+L}=\min _{1 \leq i \leq m}\left\{q_{i j}^{L}\right\}, \quad j=1, \ldots, n, \\
& \operatorname{pos}_{j}^{+U}=\max _{1 \leq i \leq m}\left\{q_{i j}^{U}\right\}, \quad j=1, \ldots, n .
\end{aligned}
$$

Definition 5. Given the beneficial and dimensionless interval decision matrix $\widetilde{Q}=\left[\widetilde{q}_{i j}\right]_{m \times n}$, the superiority degree of attribute value $\widetilde{q}_{i j}$ to the base interval $\operatorname{pos}_{j}^{+}=\left[\operatorname{pos}_{j}^{+L}, \operatorname{pos}_{j}^{+U}\right]$ for attribute $c_{j}$ is defined as

$$
b_{i j}=\frac{q_{i j}^{L}-\operatorname{pos}_{j}^{+L}}{\operatorname{pos}_{j}^{+U}-\operatorname{pos}_{j}^{+L}}+0.5 \frac{q_{i j}^{U}-q_{i j}^{L}}{\operatorname{pos}_{j}^{+U}-\operatorname{pos}_{j}^{+L}}, \quad i=1, \ldots, m, j=1, \ldots, n,
$$

where $\operatorname{pos}_{j}^{+L}$ and $\operatorname{pos}_{j}^{+U}$ are defined in (20) and (21), respectively, $j=1, \ldots, n$.

Thus, by means of formula (22), the interval decision matrix $\widetilde{Q}=\left[\widetilde{q}_{i j}\right]_{m \times n}$ is transformed into a single-point one, denoted as $B=\left[b_{i j}\right]_{m \times n}$. Based on $B=\left[b_{i j}\right]_{m \times n}$, entropy method can be used to calculate the attribute weight vector $[9,27]$, denoted as $w^{\prime \prime}=\left(w_{1}^{\prime \prime}, w_{2}^{\prime \prime}, \ldots, w_{n}^{\prime \prime}\right)$.

3.3. Determine Attribute Weights Based on Integrating Experts' Subjective Preference Information with Decision Matrix. In order to determine the attribute weights that reflect both the experts' subjective preference information on attributes and the decision matrix, the following integration method is adopted:

$$
w=\eta^{\prime} w^{\prime}+\eta^{\prime \prime} w^{\prime \prime},
$$

where $w^{\prime}$ is the attribute weight vector that is derived from the experts' subjective preference information in Section 3.1 , and $w^{\prime \prime}$ is the attribute weight vector that is derived from the interval decision matrix in Section 3.2. $\eta^{\prime}$ and $\eta^{\prime \prime}$ denote the relative importance of the experts' subjective preference information and the interval decision matrix, respectively. 
Definition 6. Given the decision matrix $B=\left[b_{i j}\right]_{m \times n}$, the positive ideal point for attribute $c_{j}$ is defined as

$$
b_{j}^{+}=\max _{1 \leq i \leq m}\left\{b_{i j}\right\}, \quad j=1, \ldots, n .
$$

Therefore, based on definition 6 , the positive ideal alternative $S^{+}$is obtained as $\left(b_{1}^{+}, b_{2}^{+}, \ldots, b_{n}^{+}\right)$. In addition, based on decision matrix $B=\left[b_{i j}\right]_{m \times n}$, the weighted sum of the deviations of alternative $\tilde{q}_{i j}$ from the positive ideal alternative $S^{+}$is obtained as

$$
r_{i}^{+}=\sum_{j=1}^{n} w_{j}\left(b_{j}^{+}-b_{i j}\right), \quad i=1, \ldots, m .
$$

It is obvious that the smaller the deviation from the positive ideal alternative $S^{+}$is, the better the alternative is. Thus, the following optimization model is set up to determine the attribute weights:

$$
\min r^{+}=\left(r_{1}^{+}, r_{2}^{+}, \ldots r_{m}^{+}\right) .
$$

Actually, model (26) is a multiobjective optimization model. Since there is no preference against the alternatives, in other words, the alternatives compete fairly, therefore, model (26) can be transformed into a singleobjective one:

$$
\min Z^{+}=\sum_{i=1}^{m} \sum_{j=1}^{n}\left(b_{j}^{+}-b_{i j}\right)\left(\eta^{\prime} w^{\prime}+\eta^{\prime \prime} w^{\prime \prime}\right)
$$

such that

$$
\begin{gathered}
\eta^{\prime 2}+\eta^{\prime \prime 2}=1, \\
0 \leq \eta^{\prime} \leq 1,0 \leq \eta^{\prime \prime} \leq 1 .
\end{gathered}
$$

Due to the limit of space, the process of solving models (27a)-(27c) is omitted. Denote the optimal solutions to models (27a)-(27c) as:

$$
\eta^{\prime *}=\frac{\sum_{i=1}^{m} \sum_{j=1}^{n}\left(b_{j}^{+}-b_{i j}\right) w_{j}^{\prime}}{\sqrt{\left[\sum_{i=1}^{m} \sum_{j=1}^{n}\left(b_{j}^{+}-b_{i j}\right) w_{j}^{\prime}\right]^{2}+\left[\sum_{i=1}^{m} \sum_{j=1}^{n}\left(b_{j}^{+}-b_{i j}\right) w_{j}^{\prime \prime}\right]^{2}}},
$$

$$
\eta^{\prime \prime}=\frac{\sum_{i=1}^{m} \sum_{j=1}^{n}\left(b_{j}^{+}-b_{i j}\right) w_{j}^{\prime \prime}}{\sqrt{\left[\sum_{i=1}^{m} \sum_{j=1}^{n}\left(b_{j}^{+}-b_{i j}\right) w_{j}^{\prime}\right]^{2}+\left[\sum_{i=1}^{m} \sum_{j=1}^{n}\left(b_{j}^{+}-b_{i j}\right) w_{j}^{\prime \prime}\right]^{2}}} .
$$

Normalize the solutions in (28) and (29) so that their sum is equal to 1 and the attribute weight vector $w^{*}$ can be obtained as:

$$
w^{*}=\eta^{\prime *} w^{\prime}+\eta^{\prime \prime *} w^{\prime \prime}
$$

\section{Illustrations}

One investment company intends to evaluate five alternatives $\left(S_{1}, S_{2}, S_{3}, S_{4}\right.$, and $\left.S_{5}\right)$ [26]. The attributes adopted are
TABLE 2: The interval attribute values in the decision matrix [22].

\begin{tabular}{ccccc}
\hline & $C_{1}$ & $C_{2}$ & $C_{3}$ & $C_{4}$ \\
\hline$S_{1}$ & {$[5,7]$} & {$[4,6]$} & {$[4,6]$} & {$[0.4,0.8]$} \\
$S_{2}$ & {$[10,12]$} & {$[6,8]$} & {$[5,7]$} & {$[1,2]$} \\
$S_{3}$ & {$[5,6]$} & {$[4,6]$} & {$[3,5]$} & {$[0.4,0.8]$} \\
$S_{4}$ & {$[9,11]$} & {$[5,7]$} & {$[5,7]$} & {$[1,1.5]$} \\
$S_{5}$ & {$[6,8]$} & {$[3,4]$} & {$[3,4]$} & {$[0.5,1]$} \\
\hline
\end{tabular}

investment amount $c_{1}$, expected net present value $c_{2}$, risk profitability value $c_{3}$, and risk loss value $c_{4}$. The attribute values of the alternatives are all interval numbers, as stated in Table 2 [26].

Among the four attributes, the expected net present value $c_{2}$ and risk profitability value $c_{3}$ are for benefits, as investment amount $c_{1}$ and risk loss value $c_{4}$ are for costs. Four experts are invited for evaluating the alternatives by providing their preference information against the attributes, as stated in Table 1.

Firstly, normalize the experts' subjective preference information in Table 1 and aggregate them into the group fuzzy preference relations on the attributes as follows:

$$
p=\left(\begin{array}{llll}
0.5000 & 0.3833 & 0.6722 & 0.2587 \\
0.6167 & 0.5000 & 0.7944 & 0.3770 \\
0.3278 & 0.2056 & 0.5000 & 0.1250 \\
0.7413 & 0.6247 & 0.8750 & 0.5000
\end{array}\right)
$$

Secondly, consider the inequality constraints among attributes $c_{2}$ and $c_{3}$, i.e., $w_{2}>w_{3}$ in models (18a) $-(18 \mathrm{~d})$. Then, based on the experts' subjective preference information, the attribute weight vector can be obtained as $w^{\prime}=(0.2237$, $0.2101,0.3126,0.2536)$.

Then, normalize the attribute values in the interval decision matrix into dimensionless ones, and the results are stated in Table 3 [26].

Furthermore, the normalized interval decision matrix is transformed into a single-point one $B=\left[b_{i j}\right]_{m \times n}$ as follows:

$$
B=\left(\begin{array}{llll}
0.6843 & 0.3900 & 0.4684 & 0.5867 \\
0.1334 & 0.6814 & 0.6397 & 0.1653 \\
0.7421 & 0.3900 & 0.2972 & 0.5867 \\
0.1924 & 0.5358 & 0.6397 & 0.1817 \\
0.4975 & 0.1593 & 0.1957 & 0.4134
\end{array}\right) .
$$

Based on decision matrix $B$, by means of entropy method, the attribute weight vector can be obtained as $w^{\prime \prime}=(0.3637,0.1891,0.1831,0.2640)$.

In addition, by means of solving models (27a)-(27c), the relative importances of the experts' subjective preference information and the interval decision matrix are obtained as follows: $\eta^{\prime *}=0.6874$ and $\eta^{\prime \prime *}=0.7263$, respectively. After normalizing $\eta^{\prime *}$ and $\eta^{\prime \prime *}$ into 0.4862 and 0.5138 , the comprehensive attribute weight vector can be obtained as $w^{*}=(0.2956,0.1993,0.2461,0.2589)$. 
TABLE 3: The normalized attribute values in the interval decision matrix.

\begin{tabular}{lcccc}
\hline & $C_{1}$ & $C_{2}$ & $C_{3}$ & $C_{4}$ \\
\hline \multirow{2}{*}{$S_{1}$} & {$[0.1837$,} & {$[0.1290$,} & {$[0.1379$,} & {$[0.1389$,} \\
& $0.3285]$ & $0.2727]$ & $0.3000]$ & $0.5357]$ \\
$S_{2}$ & {$[0.1071$,} & {$[0.1936$,} & {$[0.1724$,} & {$[0.0556$,} \\
& $0.1643]$ & $0.3636]$ & $0.3500]$ & $0.2143]$ \\
\multirow{3}{*}{$S_{3}$} & {$[0.2143$,} & {$[0.1290$,} & {$[0.1035$,} & {$[0.1389$,} \\
& $0.3285]$ & $0.2727]$ & $0.2500]$ & $0.5357]$ \\
$S_{4}$ & {$[0.1169$,} & {$[0.1613$,} & {$[0.1724$,} & {$[0.0714$,} \\
& $0.1825]$ & $0.3182]$ & $0.3500]$ & $0.2143]$ \\
$S_{5}$ & {$[0.1607$,} & {$[0.0968$,} & {$[0.1035$,} & {$[0.1111$,} \\
& $0.2738]$ & $0.1818]$ & $0.2000]$ & $0.4286]$ \\
\hline
\end{tabular}

\section{Conclusions}

This paper proposes an approach to determining the attribute weights by integrating the interval decision matrix with experts' subjective preference information on attributes in the formats of preference orderings, linguistic terms, interval numbers, and inequality constraints among partial attributes. Preference normalization and aggregation are conducted firstly. Based on the obtained group fuzzy preference relation on the attributes, the optimization models (18a)(18d) are set up to calculate the subjective weights, while satisfying the inequality constraints among partial attributes. The objective attribute weights are obtained by using entropy method after normalizing the interval decision matrix. The subjective weights and the objective weights are integrated in the optimization models (27a)-(27c) in order to calculate the relative importance of the subjective information and the objective information.

The attribute weights, obtained in the integration models (27a)-(27c), take into account experts' subjective preference information and the objective information of the decision matrix so that they are more reasonable and credible. In addition, this paper enables experts to express their preference information in the easiest ways and accurately, especially in fuzzy or uncertain decision environment. Compared with the current research, the proposed approach has more universal significance and practical application prospect.

\section{Data Availability}

The data used to support the findings of this study are available from the corresponding author upon request.

\section{Conflicts of Interest}

The authors declare no conflict of interest regarding the publication of this paper.

\section{Acknowledgments}

This work was financially supported by the Natural Science Foundation of Liaoning Province (Grant no. 2013020022).

\section{References}

[1] S. J. Chen and C. L. Hwang, Fuzzy Multiple Attribute Decision Making: Methods and Applications, Springer-Verlag, New York, NY, USA, 1992.

[2] S. M. Chen, "Fuzzy group decision making for evaluating the rate of aggregative risk in software development," Fuzzy Sets and Systems, vol. 118, no. 1, pp. 75-88, 2001.

[3] S. Liu, F. T. S. Chane, and W. X. Ran, "Multi-attribute group decision-making with multi-granularity linguistic assessment information: an improved approach based on deviation and TOPSIS," Applied Mathematical Modelling, vol. 37, no. 24, pp. 10129-10140, 2013.

[4] S. M. Chen and S. J. Niou, "Fuzzy multiple attributes group decision-making based on fuzzy preference relations," Expert Systems with Applications, vol. 38, no. 4, pp. 3865-3872, 2011.

[5] R. X. Liang, J. Q. Wang, and H. Y. Zhang, "A multi-criteria decision-making method based on single-valued trapezoidal neutrosophic preference relations with complete weight information," Neural Computing and Applications, vol. 2, pp. 1-16, 2017.

[6] J. Ma, Z. P. Fan, and L. H. Huang, "A subjective and objective integrated approach to determine attribute weights," European Journal of Operational Research, vol. 112, no. 2, pp. 397-404, 1999.

[7] Z. S. Xu, "Multiple-attribute group decision making with different formats of preference information on attributes," IEEE Transactions on Systems, Man, and Cybernetics, Part B: Cybernetics, vol. 37, no. 6, pp. 1500-1511, 2007.

[8] V. Mattila and K. Virtanen, "Ranking and selection for multiple performance measures using incomplete preference information," European Journal of Operational Research, vol. 242, no. 2, pp. 568-579, 2015.

[9] M. Alemi-Ardakani, A. S. Milani, S. Yannacopoulos et al., "On the effect of subjective, objective and combinative weighting in multiple criteria decision making: A case study on impact optimization of composites," Expert Systems with Applications, vol. 46, pp. 426-438, 2016.

[10] H. B. Kong, Weights and Preference Analysis in MultiAttribute Group Decision-Making, Yunnan University, Kunming, China, 2007.

[11] P. Cheng and W. Liu, "A Method of determining attribute weights based on subjective preferences in multi-attribute group decision making," Control and Decision, vol. 25, no. 11, pp. 1645-1656, 2010.

[12] Y. M. Wang and C. Parkan, "A general multiple attribute decision-making approach for integrating subjective preferences and objective information," Fuzzy Sets and Systems, vol. 157, no. 10, pp. 1333-1345, 2006.

[13] L. C. Cheng and H. A. Wang, "A fuzzy recommender system based on the integration of subjective preferences and objective information," Applied Soft Computing, vol. 18, pp. 290-301, 2014.

[14] G. G. Lin and H. Z. Song, "A multi-attribute group decision making, based on integrating subjective and objective weight information," Statistics and Decision Making, vol. 12, pp. 70-73, 2016.

[15] J. Li, J. Q. Wang, and J. H. Hu, "Multi-criteria decisionmaking method based on dominance degree and BWM with probabilistic hesitant fuzzy information," International Journal of Machine Learning and Cybernetics pp. 1-15, 2018, In press.

[16] F. Franceschini, D. Maisano, and L. Mastrogiacomo, “A new proposal for fusing individual preference orderings by rank- 
ordered agents: a generalization of the Yager's algorithm," European Journal of Operational Research, vol. 249, no. 1, pp. 209-223, 2016.

[17] R. X. Nie, Z. P. Tian, X. K. Wang, J. Q. Wang, and T. L. Wang, "Risk evaluation by FMEA of supercritical water gasification system using multi-granular linguistic distribution assessment," Knowledge-Based Systems, 2018, In press.

[18] Z. S. Xu, "A method for multiple-attribute decision making with imcomplete weight information in linguistic setting," Knowledge-Based Systems, vol. 20, no. 8, pp. 719-725, 2007.

[19] W. E. Yang and J. Q. Wang, "Multi-criteria semantic dominance: a linguistic decision aiding technique based on incomplete preference information," European Journal of Operational Research, vol. 231, no. 1, pp. 171-181, 2013.

[20] Z. P. Tian, J. Q. Wang, and H. Y. Zhang, “An integrated approach for failure mode and effects analysis based on fuzzy best-worst, relative entropy, and VIKOR methods," Applied Soft Computing, 2018, In press.

[21] S. Liu, TS Chan Felix, and W. X. Ran, "Decision making for the selection of cloud vendor: an improved approach under group decision-making with integrated weights and objective/ subjective attributes," Expert Systems with Applications, vol. 55, pp. 37-47, 2016.

[22] N. Yang, X. W. Liao, and W. W. Huang, "Decision support for preference elicitation in multi-attribute electronic procurement auctions through an agent-based intermediary," Decision Support Systems, vol. 57, no. 1, pp. 127-138, 2014.

[23] P. B. Sun, Y. T. Liu, X. Z. Qiu et al., "Hybrid multiple attribute group decision-making for power system restoration," Expert Systems with Applications, vol. 42, no. 19, pp. 6795-6805, 2015.

[24] F. Herrera, L. Martinez, and P. J. Sanchez, "Managing non-homogeneous information in group decision making," European Journal of Operational Research, vol. 166, no. 1, pp. 115-132, 2005.

[25] Z. S. Xu, "Goal planning model for linguistic multi-attribute decision-making," Journal of Management Science, vol. 9, no. 2, pp. 9-17, 2006.

[26] R. Lan, "An ideal-point method of multiple attribute decision making based on interval number distance," Xi'an University of Posts and Telecommunications, vol. 14, no. 1, pp. 153-156, 2009.

[27] Y. Ji, G. H. Huang, and W. Sun, "Risk assessment of hydropower stations through an integrated fuzzy entropyweight multiple criteria decision making method: a case study of the Xiangxi River," Expert Systems with Applications, vol. 42 , no. 12, pp. 5380-5389, 2015. 


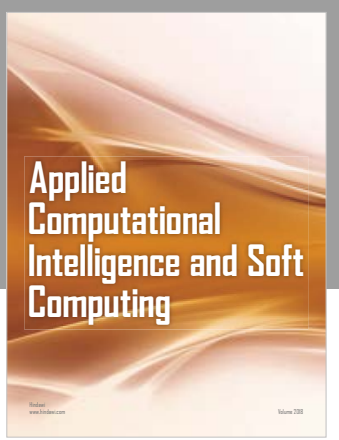

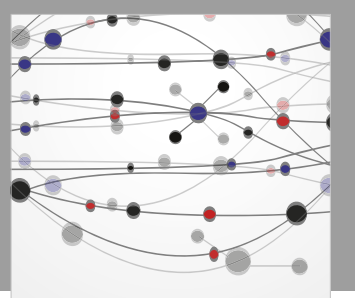

The Scientific World Journal
Submit your manuscripts at

Computing
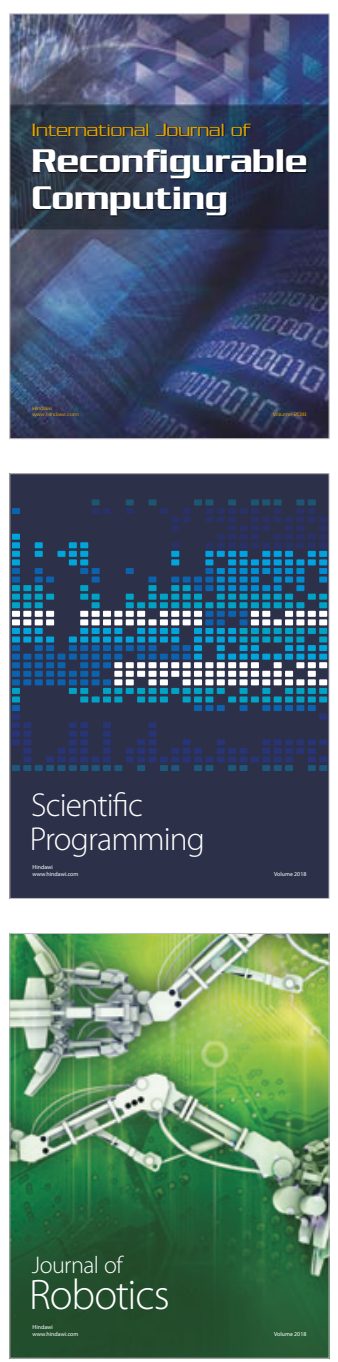

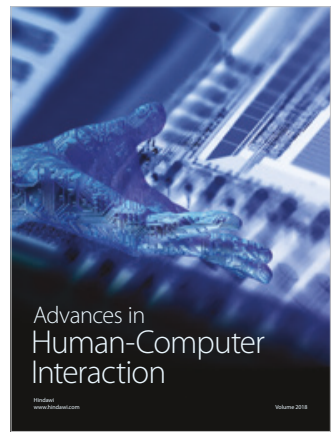

Human-Compute

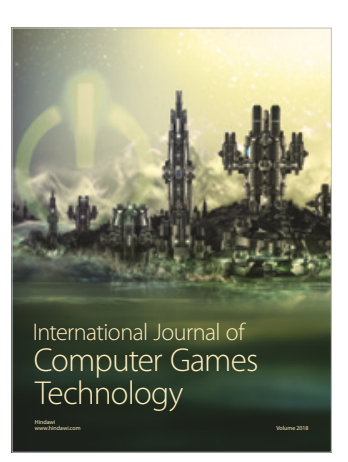

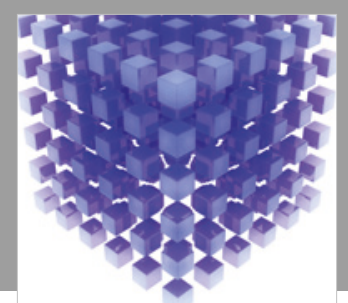

Mathematical Problems in Engineering

\section{Engincering}
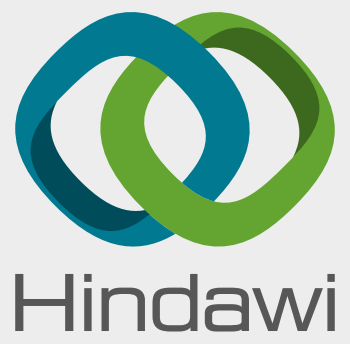

www.hindawi.com
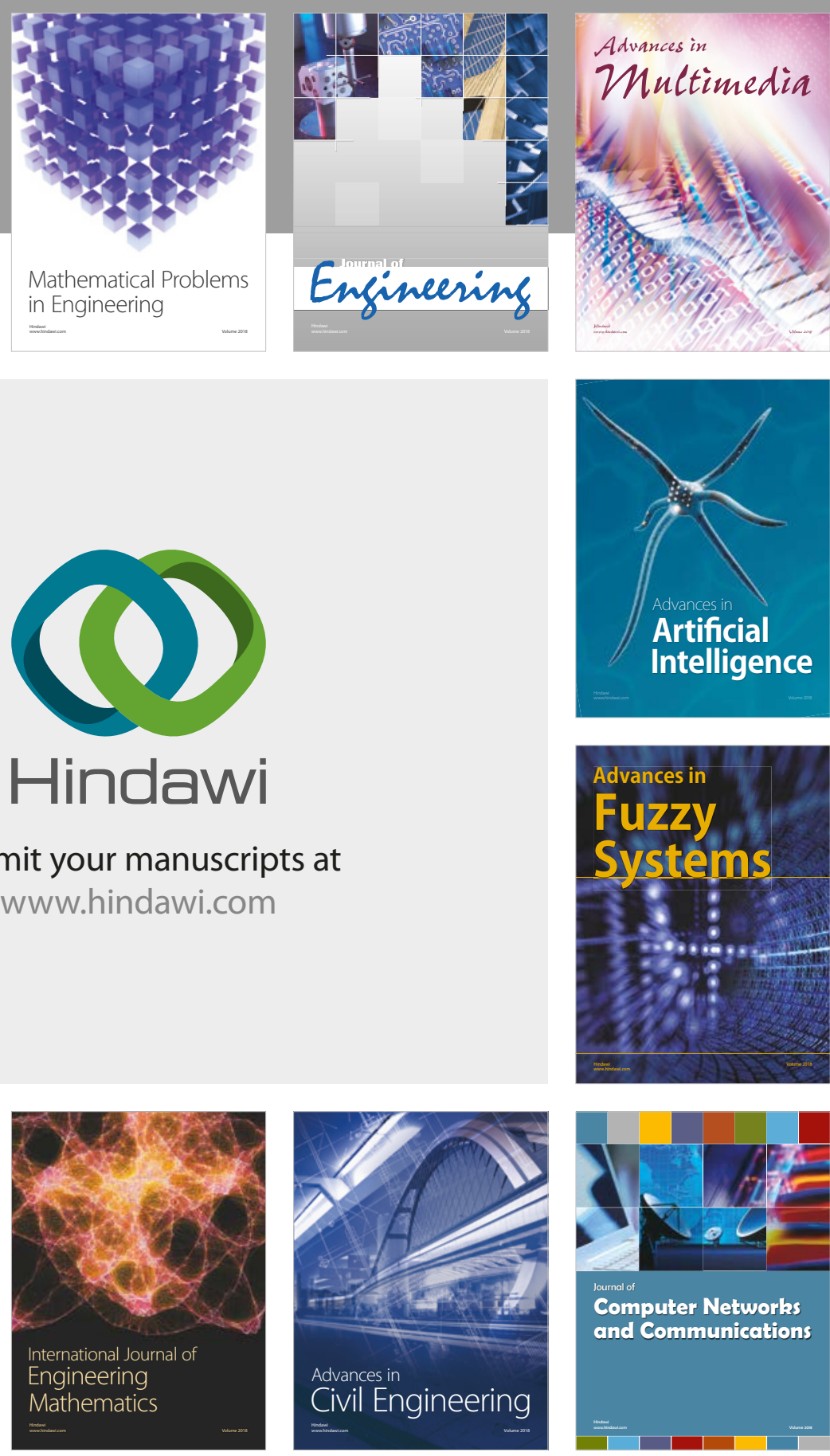

Computer Networks and Communications

Multimedia
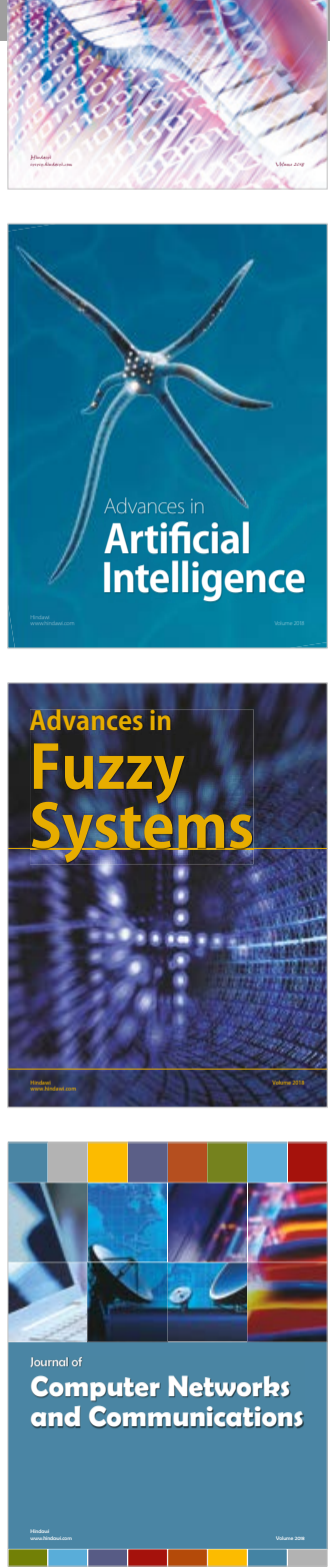

Advances in

Modelling \&

Simulation

in Engineering

interaction

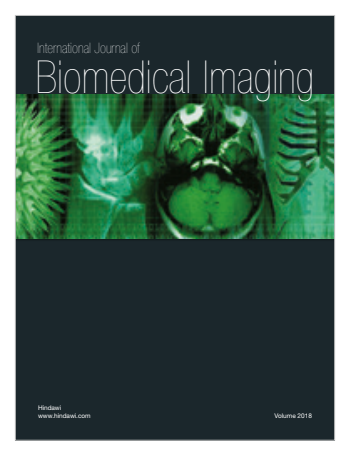

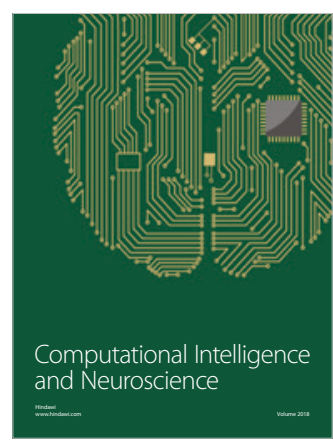

